\title{
Recognition of two-phase flow pattern inside evaporator tubes
}

\author{
Amedeo Amoresano ${ }^{1}$, Paolo Iodice ${ }^{1}$, Giuseppe Langella ${ }^{1, *}$, Vincenzo Niola ${ }^{1}$ and Giuseppe \\ Quaremba $^{1}$ \\ ${ }^{1}$ University "Federico II" of Naples, Department of Industrial Engineering, 80125 Naples, Italy
}

\begin{abstract}
The convective heat exchange mechanism in two-phase regime, can allow high exchange coefficients, as long as particular conditions are respected, in terms of vapor quality. In evaporator tubes this problem is particularly important as a deviation from the optimal flow conditions can drastically drop the efficiency of the heat exchange. This work describes an innovative methodology for identifying the different two-phase flow regimes in evaporator pipes, through the processing of the vibrational data acquired on the pipe itself. An experimental apparatus with transparent pipes has been built up to recognize flow patterns by a fast image acquisition camera. Images have been associated to vibration data acquired on pipes by accelerometer. The analysis of frequency spectrum, led to first interesting results thus it is possible to distinguish between "no bubbles" regimes and different "bubbles" regimes. A wide test campaign has been realized on vertical and horizontal pipes, simulating steam bubbles with nitrogen bubbles, reproducing the main flow patterns. The paper demonstrates the possibility to design smart and non-intrusive sensor to be applied on evaporated tubes for the recognizing of anomalous thermal flow condition and sets the stage for future engineering work.
\end{abstract}

\section{Introduction}

Two-phase flows, in particular those where liquid and vapor phases coexist, are present in a multitude of plant applications, both industrial and civil. Evaporators and condensers are fundamental components of refrigeration and energy systems. These flows are characterized by a rather complex fluid dynamics which makes coding them difficult by means of simple physical laws. In most cases empirical formulations based on numerous experimental findings are used. On the other hand, the characterization of specific patterns of behavior of these flows is fundamental in the design of evaporators and condensers and in their monitoring during operation. As regards the heat exchange, the two-phase flow conditions are characterized by a wide range of exchange coefficients depending on the preponderance of one phase over the other. Under the best conditions, these systems allow to achieve the highest heat exchange coefficients, of the order of $10^{4} \mathrm{~W} / \mathrm{m}^{2} \mathrm{~K}$. However, even slight deviations from the optimal conditions can cause the exchange coefficient to drop by two orders of magnitude. This is the case, for example, of the transition from the condition of nucleate boiling to that of film boiling. In some plant situations, all this can lead to considerable damage, as in the case of steam generators installed in nuclear plants. All this justifies the importance that can be assumed by monitoring the correct conditions of heat exchange in two-phase flow, to intervene promptly in case of deviation from the design parameters. In technical practice, various techniques have been developed [1], based on different physical phenomena, which allow the non-invasive monitoring of the pipes 
involved. Some of these techniques are based on the attenuation of gamma rays as a function of the vacuum degree of the liquid vapor mixture $[2,3]$. Other techniques are based on the variation of the electrical impedance of the mixture as the vacuum fraction varies $[4,5,6]$. In some cases, these techniques are associated with high-speed photography [7] in transparent experimental pipes, or with the introduction of optical fibers inside the pipe [8].The alteration of the speed of sound as a function of the vacuum fraction can be related to the specific two-phase flow pattern by means of pressure transducers [9].Even thermometry and infrared analysis can give interesting results in the detection of anomalous conditions of two-phase flow [10,11].Last but not least is the technique based on the analysis of the vibrations induced by the two-phase flow on the pipe [12]. Vibration can be decoded by identifying characteristic frequencies by Fourier analysis or used by more sophisticated statistical techniques [13].This work presents a methodology for identifying the different two-phase flow patterns starting from vibrational data acquired using accelerometers, but processed using an innovative technique based on discriminant analysis. The analysis was developed starting from a campaign of experimental surveys in different conditions of the vacuum fraction, associated with a series of fast camera shots on a transparent section of pipe. This technique may be more useful than other more mature and already in use, thanks to the fact that it is non-invasive and can identify different twophase flow patterns in real time. All this can make it possible to intervene on the system promptly, before even worse conditions and irreversible damage occur.

\section{Two-phase flow patterns}

In flow boiling (or forced convection boiling), fluid flow is forced over a surface by external means such as a pump, as well as by buoyancy effects. Therefore, flow boiling is always accompanied by other convection effects. Conditions depend strongly on geometry, which may involve external flow over heated plates and cylinders or internal (duct) flow. In nuclear reactors, most of boiling regimes are just forced convection boiling. The flow boiling is also classified as either external and internal flow boiling depending on whether the fluid is forced to flow over a heated surface or inside a heated channel. Internal flow boiling is much more complicated in nature than external flow boiling because there is no free surface for the vapor to escape, and thus both the liquid and the vapor are forced to flow together. The two-phase flow in a tube exhibits different flow boiling regimes, depending on the relative amounts of the liquid and the vapor phases. The Fig. 1 shows the two-phase flow regimes inside a vertical pipe. 


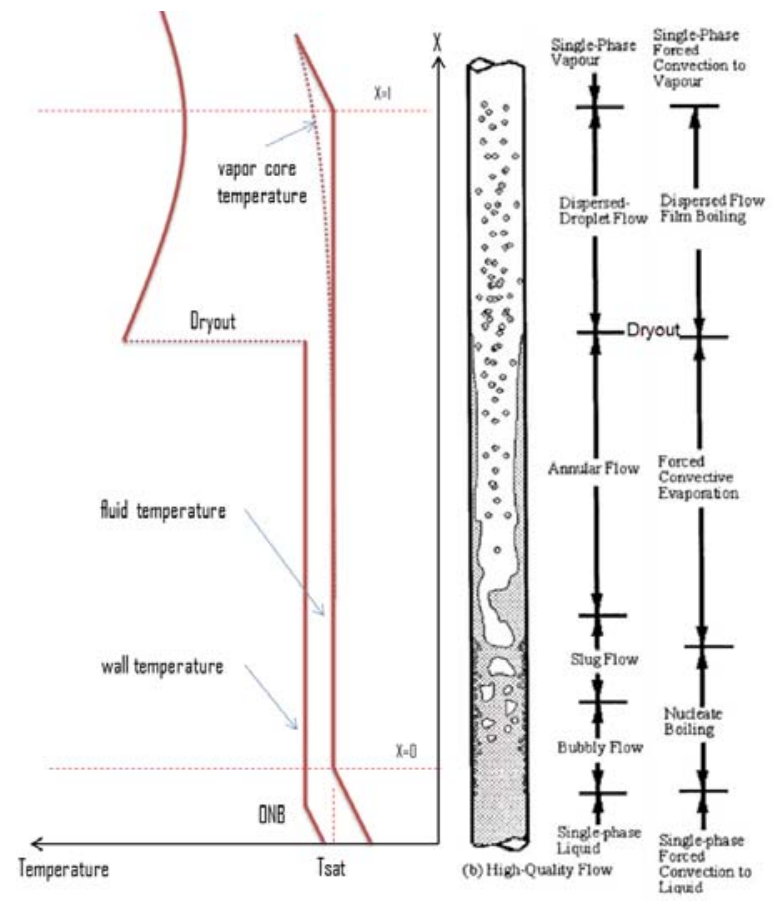

Fig. 1. Two phase flow patterns [14]

Typical flow boiling modes in a vertical channel are depicted in figure 1 [14]. This figure shows the typical order of the flow regimes that are encountered from inlet to outlet of a heated channel. At the inlet, the liquid enters subcooled (at the lower temperature than saturation). In this region the flow is single-phase. As the liquid heats up, the wall temperature correspondingly rises. As the wall temperature exceeds the saturation temperature (e.g. $285^{\circ} \mathrm{C}$ at $6.8 \mathrm{MPa}$ ), subcooled nucleate boiling begins. Bubbles nucleate in the superheated thermal boundary layer on the heated wall but tend to condense in the subcooled bulk.

Further increase in liquid temperature causes the liquid bulk to reach its saturation temperature and the convective boiling process to pass through the bubbly flow into the slug flow. Increasing void fraction causes the structure of the flow to become unstable. The boiling process passes through the slug and churn flow into the annular flow regime with its characteristic annular film of the liquid. At given combinations of flow rate through a channel, pressure, flow quality, and linear heat rate, the wall liquid film may run out and the wall may be dried out. At the dryout point the wall temperature significantly rises in order to dissipate the applied heat flux. The post-dryout flow (mist or drop flow) in the heated channel is undesirable because the presence of such flow regime is accompanied with significantly higher wall temperatures and high fluctuation of wall temperatures.

That said, it highlights how important it is to know the behavior of the bubbles, their size, their distribution in order to obtain a high temperature flow and avoid reaching the thermal crisis pendants. Obviously, what has been said applies to all types of liquids. The same problems are encountered for HFCs and for oils used as heat transfer fluid. 


\section{Material and methods}

\subsection{Experimental Set-Up}

An "ad hoc" experimental facility has been and designed and built up. A scheme of the design is shown in Figure 2.

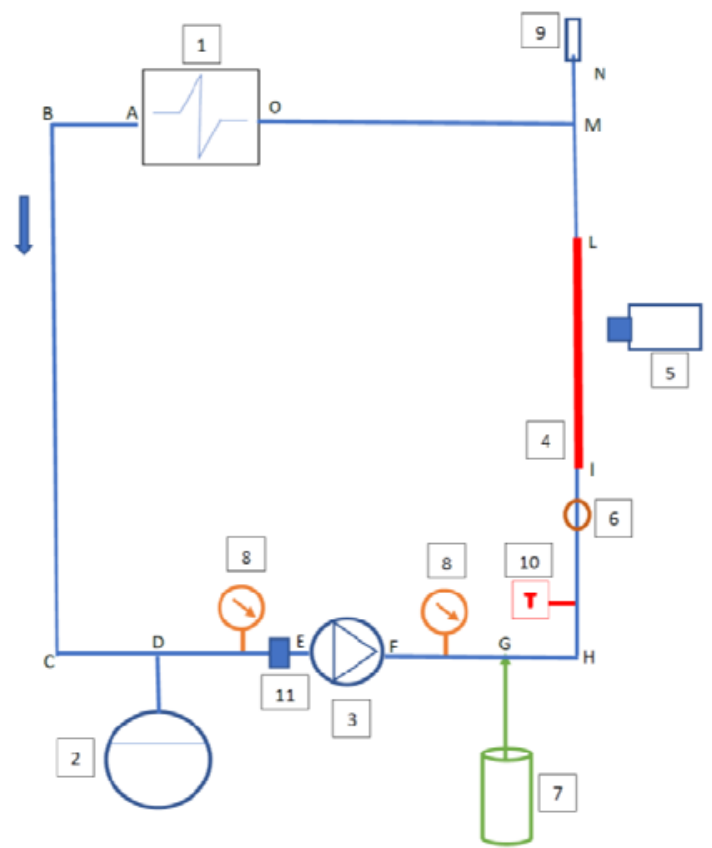

Fig. 2. Experimental set-up

The facility contains an electric water heater (1) with capacity of 5 liters controlled by a PID to stabilize the temperature (the range is between external temperature and $100{ }^{\circ} \mathrm{C}$ ). The item $\mathrm{AB}$ is a first part of the duct that has been joint by the first $90^{\circ}$ connection with the down tube $\mathrm{BC}$. At the end of the section $\mathrm{BC}$ another $90^{\circ}$ connection joints the pipe with the expansion vessel (2). The Expansion vessel is able to give the requested pressure to the pipe and to recover and stabilized the movements of the fluid. It is important to highlight that, all measurements devices and components. are placed considering the steady conditions of the flow and so the mutual distance between a component and the measurement points is always bigger than 5 diameters. On the pipeline, section DE, takes place a volumetric flow meter and a piezoelectric sensor able to measure the inlet pressure of the centrifugal pump and on the section FG. another piezoelectric sensor. measures the outlet pressure. The $\mathrm{G}$ point instead represents the inlet of the compressed air taken from the pressure vessel (7). This air, according with the pressure and temperature of the liquid phase, will generate a bubbles cluster of defined shape and size. The new two phase fluid, will pass through the 90 connection, point $\mathrm{H}$ of the pipe, and will be routed to the vertical pipe. The vertical pipe is the track where the requested measurements will carried out. On the section HI will be measured the temperature of the two phase fluid and immediately 
after an accelerometer will be placed to record the vibration due to the bubbles presence. The following section of the pipe IL will be in transparent material (plexiglass or other materials) to verify which kind of two phase flow has been developed. The pipe continues with the section LM where a T joint permits to mount an air vent (9) to drain the gas blown. Section MO brings the water back into the heater. The whole pipe is insulated to try have constant temperature

\subsection{Test conditions and data acquisition}

The experimental setup is able to inject gas (in this case nitrogen) and create a simulated two-phase flow pattern similar to what happens in steam generators. The aim of the work is to determine, by means of non-intrusive measures, which is the type of bubble motion that develops inside the tube. To determine this, accelerometers are used that are capable to measure the vibrations induced by the bubbles passing through the duct. The tests reported below are carried out in two configurations. The first involves only the passage of the liquid phase without jars at a certain pressure and at room temperature. The second instead involves the injection of a known volumetric flow rate of gas (nitrogen) into the duct. For both configurations, the vibrations along two axes ( $\mathrm{X}$ and $\mathrm{Z}$ ) are measured according to the orientation of the axes in figure 2 and then their pattern is determined.

Test conditions are reported in the table below.

Table 1. Test conditions

\begin{tabular}{|l|l|}
\hline Pump Volumetric Flow Rate & $301 / \mathrm{min}$ \\
\hline Pipe pressure & $2 \mathrm{~atm}$ \\
\hline $\mathrm{N}_{2}$ volumetric flow rate & $0-2 \mathrm{Nl} / \mathrm{min}$ \\
\hline Water Temperature & $23^{\circ} \mathrm{C}$ \\
\hline Test duration for each configuration & $30 \mathrm{sec}$ \\
\hline Sampling frequency & $20.000 \mathrm{~Hz}$ \\
\hline
\end{tabular}

The tests were carried out on two conditions:

The first without gas blowing to evaluate only the vibration of the system with circulating water.The second with gas blowing (nitrogen) under the conditions reported in the data to evaluate the behavior of the two-phase flow. 

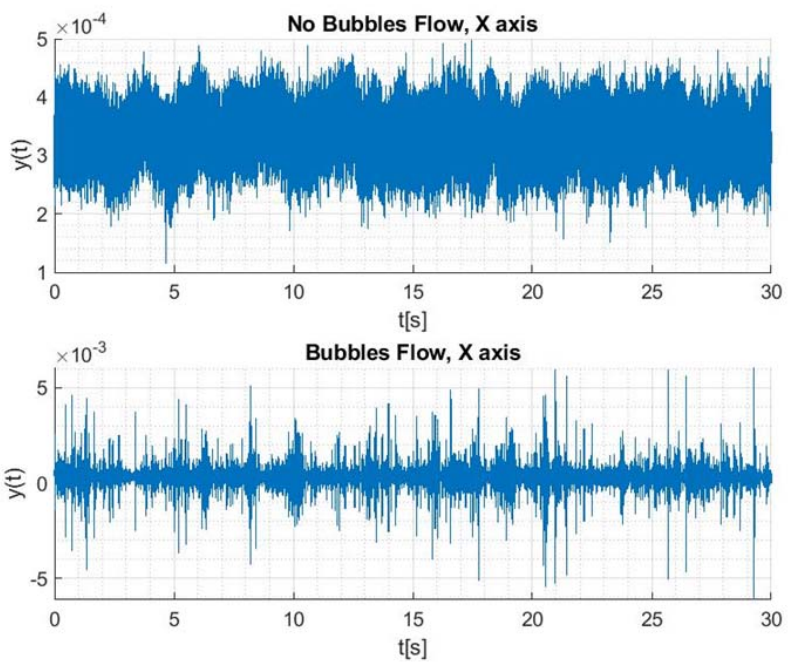

Fig. 3. Vibration along $\mathrm{X}$ axis
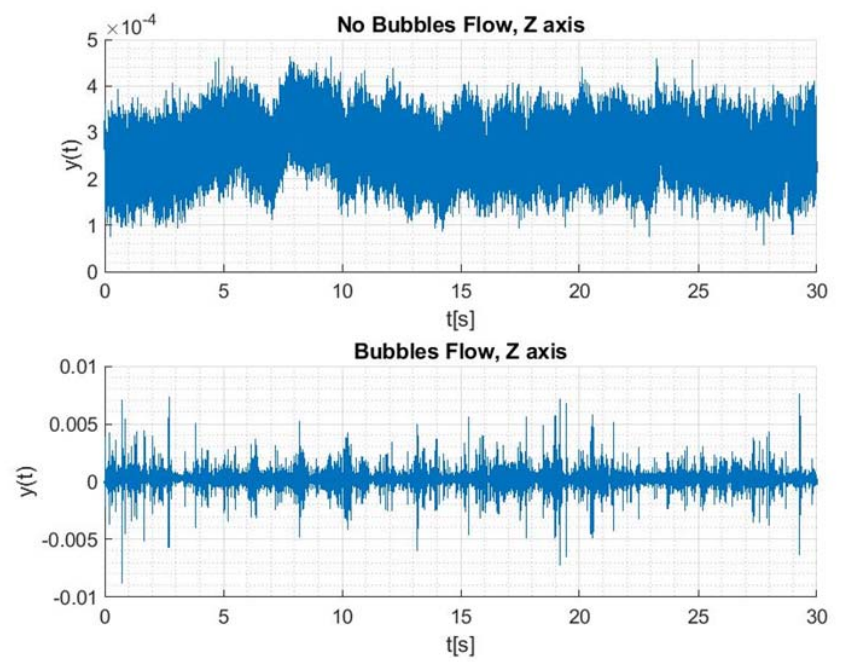

Fig. 4. Vibration along $\mathrm{Z}$ axis

Figures 3 and 4 describe the accelerometer signals along $\mathrm{X}$ and $\mathrm{Z}$ axis, comparing the case with no bubble with the bubble one. No bubble case has a base line with a width between 2 and $5 \times 10-4$ along $\mathrm{x}$ and $\mathrm{z}$ axis. This spectrum is generated by the whole system and the running stream water. The first match between the signals put in evidence that the order of amplitude is quite different. The presence of the bubbles modifies intensity of the vibration mode of the system both along $\mathrm{x}$ and along $\mathrm{z}$ axis. 


\section{PROCESSING OF RESULTS}

Vibrational data have been processed by discriminant analysis. Similar applications of this innovative technique have also been realized for the characterization of stationary sprays (gas turbines) [15] and stationary (internal combustion engines) [16,17]. This technique goes beyond the classic identification of characteristic frequencies through Fourier analysis, applying the statistical theory of chaos to identify and characterize particular operating conditionsTo apply this method it is necessary to consider that the signal is the sum of 30 signal of 1 second. In this way we have, for each acquisition, 30 strips of 1 second and, due to the sampling frequency of $20.000 \mathrm{~Hz}$, each acquisition will have 20.000 points. This procedure aims to evaluate all thirty acquisitions among themselves. The evaluation of the behavior of the signal needs several independent variables (variables that must be orthonormal among them) because they must describe their average convergence to zero. If this happens, the variables that tend to zero are able to describe the statistical behavior of the acquired signal. In the following table the first column describes 12 independent statistical variables.

Table 2. Test of Equality of Groups Means

Tests of Equality of Group Means
\begin{tabular}{|l|r|r|r|r|r|}
\hline \multicolumn{1}{|c|}{$\begin{array}{c}\text { Wilks' } \\
\text { Lambda }\end{array}$} & \multicolumn{1}{c|}{$\mathrm{F}$} & \multicolumn{1}{c|}{ df1 } & \multicolumn{1}{c|}{ df2 } & \multicolumn{1}{c|}{ Sig. } \\
\hline FORM_FACTOR &, 784 & 10,646 & 3 & 116 &, 000 \\
SQUUARED_OSCILLATIO &, 126 & 268,848 & 3 & 116 &, 000 \\
N_INDEX & $\mathrm{a}$ & & & & \\
SYNCHRONY_INDEX &. & & & & \\
PEARSON_CORRELATIO & & & & & \\
N &, 506 & 37,729 & 3 & 116 &, 000 \\
KURTOSIS &, 938 & 2,543 & 3 & 116 &, 060 \\
SKEWNESS &, 261 & 109,365 & 3 & 116 &, 000 \\
RMS &, 261 & 109,365 & 3 & 116 &, 000 \\
RSS &, 156 & 208,904 & 3 & 116 &, 000 \\
CREST_FACTOR &, 074 & 484,427 & 3 & 116 &, 000 \\
BETA_OSCILLATION_IN &, 340 & 75,046 & 3 & 116 &, 000 \\
DEX &, 332 & 77,910 & 3 & 116 &, 000 \\
SHANNON_ENTROPY & & & & \\
LOG_ENTROPY &
\end{tabular}

a. Cannot be computed because this variable is a constant.

The tests of equality of group means measure each independent variable's potential before the model is created. Each test displays the results of a one-way ANOVA for the independent variable using the grouping variable as the factor. If the significance value is greater than 0.10 , the variable probably does not contribute to the model. According to the results in this table, every variable (except Skewness) in the discriminant model is significant. In addition to measures, the Discriminant Analysis procedure provides the eigenvalues and Wilks' lambda tables for seeing how well the discriminant model, as a whole, fits the data. The eigenvalues table provides information about the relative efficacy of each discriminant function. 
Table 3. Eigenvalues of analysis functions

\begin{tabular}{|l|r|r|r|r|}
\multicolumn{5}{c|}{ Eigervalues } \\
\hline Function & Eigenvalue & \% of Variance & Cumulative \% & $\begin{array}{c}\text { Canonical } \\
\text { Correlation }\end{array}$ \\
\hline 1 & $26,770^{\mathrm{a}}$ & 79,1 & 79,1 &, 982 \\
2 & $4,833^{\mathrm{a}}$ & 14,3 & 93,4 &, 910 \\
3 & $2,231^{\mathrm{a}}$ & 6,6 & 100,0 &, 831 \\
\hline
\end{tabular}

a. First 3 canonical discriminant functions were used in the analysis.

Wilks' lambda is a measure of how well each function separates cases into groups. It is equal to the proportion of the total variance in the discriminant scores not explained by differences among the groups. Smaller values of Wilks' lambda indicate greater discriminatory ability of the function.

Table 4. Wilk's Lambda coefficient

\begin{tabular}{|l|r|r|r|r|}
\multicolumn{1}{|c|}{ Wilks' Lambda } \\
\hline Test of Function(s) & \multicolumn{1}{|c|}{$\begin{array}{c}\text { Wilks' } \\
\text { Lambda }\end{array}$} & Chi-square & \multicolumn{1}{c|}{ df } & \multicolumn{1}{c|}{ Sig. } \\
\hline 1 through 3 &, 002 & 713,668 & 18 &, 000 \\
2 through 3 &, 053 & 334,738 & 10 &, 000 \\
3 &, 310 & 133,688 & 4 &, 000 \\
\hline
\end{tabular}

The associated chi-square statistic tests the hypothesis that the means of the functions listed are equal across groups. The small significance value indicates that the discriminant function does better than chance at separating the groups.

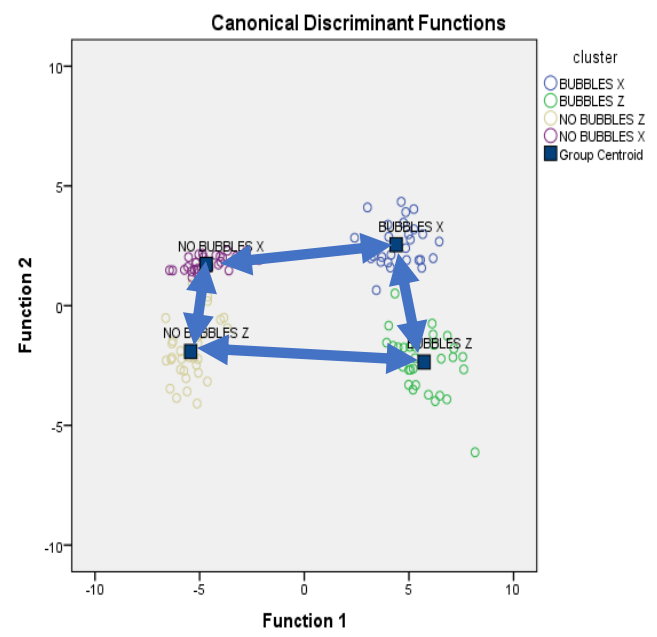

Fig. 5. Phase diagram

Finally the phase diagram of the most significant functions, reported in fig. 5 shows how the case relating to the passage of the bubbles is clearly distinguishable. This methodology 
therefore appears promising also for distinguishing between different modes of bubble motion. Ultimately, this analysis can be used to analyze the two-phase flow inside a pipe in a non-intrusive and non-visual way.

\section{CONCLUSION}

Using Discriminant Analysis, we created a model that classifies run as cluster 1, 2, 3 or 4. The model is able to verify the behavior of several index. Once independent statistical variables have been defined, it is possible to deduce indicators that analyze the variance of the data. The first is the variance able to define how each point of the acquired signal varies with respect to the other points of the same signal and thus identifies the behavior of the bubbles of the same cluster (points green and blue of the fig. 4). In addition, another indicator is deduced, the covariance. This indicator is able to define how a cluster of data behaves with respect to another cluster, highlighted by the distance between two clusters in the phase diagram (blue rows in Fig. 5). With these two indices it is therefore possible to understand, through the vibrational data, how a system, for example the bubble-free duct, behaves in totally liquid flow and how it differs when bubbles are present. It is therefore possible to define clusters that identify on a diagram that we will call phase diagrams, which are representative of the mode of vibration of the system in different configurations. In our case the configurations are given by the presence of bubbles and their absence.

The results obtained in this activity appear very interesting because they lay the foundations for the creation and development of innovative smart sensors to be used in concentrating solar plants receivers. These sensors can be very useful in the immediate diagnosis of an imminent condition of anomalous heat exchange, allowing rapid intervention to correct these anomalies and avoid costly damage to the systems. Surely, at present, there may still be some uncertainties of the method in recognizing a particular pattern with respect to another, especially for particularly similar ones. The recognition technique must be perfected in the next experiments. Further steps in this experimentation and data analysis can certainly be promising in this regard.

\section{References}

1. Nabil Ghendour, Mahmoud Meribout, Abdelwahid Azzi, Review of measurement techniques for void fraction of two-phase flow through annulus, Measurement 165 (2020), 108196

2. A.M. Osman, A.M. Abdel-monem, Measurement of void fraction in pipes by nuclear trasmission based techniques, Annals of Nuclear Energy 152 (2021) 107994

3. Yu Zhao, Qincheng Bi, Yuejin Yuan, HaicaiL, Void fraction measurement in steamwater two-phase flow using the gamma ray attenuation under high pressure and high temperature evaporating conditions, Flow Measurement and Instrumentation 49 (2016)18-3020 
4. Andrzej Krupa , Marcin Lackowski, Anatol Jaworek, Capacitance sensor for measuring void fraction in small channels, Measurement 175 (2021) 109046

5. Nikolas Libert, Rigoberto E.M.Morales, Marco Jose da Silva, Capacitive measuring system fort wo-phase flow monitoring. Part1:Hardware design and evaluation, Flow Measurement and Instrumentation 47(2016)90-99

6. Pieter Gijsenbergh, Robert Puers, Permittivity-based void fraction sensing for microfluidics, Sensors and Actuators A 195 (2013) 64- 70

7. Madhavi V. Sardeshpande, Sagar Harinarayan, Vivek V. Ranade, Void fraction measurement using electrical capacitance tomography and high speed photography, Chemical engineering research and design 94 ( $\left.2 \begin{array}{llll}2 & 0 & 1 & 5\end{array}\right)$ 1-11

8. Q.Y. Yang, N.D. Jin, L.S. Zhai, D.Y. Wang, F. Wang, Experimental study of slug and churn flows in a vertical pipe using plug-in optical fiber and conductance sensors, Experimental Thermal and Fluid Science 107 (2019) 16-28

9. Claudia Esposito, Onur Yenigun, Jean-Baptiste Gouriet, Johan Steelant,Maria Rosaria Vetrano, Void fraction and speed of sound measurement in cavitating flows by the three pressure transducers (3PT) technique, Experimental Thermal and Fluid Science 112 (2020) 109949

10. Mao Takeyama, Tomoaki Kunugi, Takehiko Yokomine, Zensaku Kawara, Phase detection aided thermometry (PDaT) for two-phase flow, International Journal of Heat and Mass Transfer 118 (2018) 492-497

11. Chao Wang, Ning Zhao, Lide Fang, Tao Zhang, Yue Feng, Void fraction measurement using NIR technology for horizontal wet-gas annular flow, Experimental Thermal and Fluid Science 76 (2016) 98-108

12. E. Laggiard and I. Runkel, Detection Of Subcooled Boiling In A Pwr Using Noise Analysis And Calculation Of The Steam Void Fraction, Ann. Nucl Energy, Vol. 24, No. I, pp. 49-54, 1997

13. Philipp Wiedemann, Alexander Döß, Eckhard Schleicher, Uwe Hampel, Fuzzy flow pattern identification in horizontal air-water two-phase flow based on wire-mesh sensor data, International Journal of Multiphase Flow 117 (2019) 153-162

14. Tong, L. S., Weisman, Joel. Thermal Analysis of Pressurized Water Reactors. Amer Nuclear Society, 3rd edition, 5/1996. ISBN-13: 978-0894480386

15. Amoresano, A., Langella, G., Di Santo, M., Iodice, P. Advanced Imaging Techniques for Multiphase Flows Analysis, Journal of Physics: Conference Series, 2017, 882(1), 012004

16. Montanaro, A., Allocca, L., Amoresano, A., Langella, G. , A dynamic System Approach for the Experimental Characterization of a Multi-Hole Spray, SAE Technical Papers, 2017

17. Allocca, L., Montanaro, A., Amoresano, A., Langella, G., Niola, V., Quaremba, G., Chaos Theory Approach as Advanced Technique for GDI Spray Analysis, SAE Technical Papers, 2017 